\title{
Preferential approach to conspecifics as a function of different rearing conditions
}

\author{
S. E. SIGMAN, D. R. LOVERN, and A. H. SCHULMAN \\ Virginia Polytechnic Institute and State University, Blacksburg, Virginia 24061
}

\begin{abstract}
Forty-six White Leghorn chicks were tested at $48 \mathrm{~h}$ posthatch to determine their ability to discriminate and preferentially respond to individual companions and strangers. In the first experiment, socially reared chicks showed no preference when allowed a simultaneous choice between either a familiar or an unfamiliar socially reared chick. In the second experiment, socially reared chicks preferred $(p=.012)$ an unfamiliar socially reared chick to an unfamiliar chick reared in isolation. In the third experiment, chicks reared in isolation preferred $(p=.036)$ a socially reared chick to an isolation-reared chick. Results indicate that chicks $48 \mathrm{~h}$ old respond to others on the basis of behavioral as well as morphological characteristics.
\end{abstract}

Adult chickens are able to recognize individual conspecifics as evidenced in the establishment of dominance hierarchies (Schelderup-Ebbe, 1935). However, the age at which social discriminations first appear and the conditions necessary for the formation of such discriminations are not well established. In an early imprinting study, Smith (1957) presented evidence that chicks 10 days old are able to discriminate flock mates from unfamiliar birds. Others have also shown that chicks recognize individuals, and begin to establish peck orders at about 18 days of age (Guhl, 1958). Since these early reports, it has become abundantly clear that chicks make social discriminations at much earlier ages. For example, Rajecki and Lake (1972) have demonstrated that DeKalb hybrid chicks dyed red, yellow, or green and housed in pairs of similar colors prefer chicks of their own color when tested $24 \mathrm{~h}$ posthatch. Chicks dyed the same color and reared in isolation exhibit no preference for one color over the other. These results confirm those of Salzen and Cornell (1968) with birds 9 days old and indicate that these neonates imprint on, and prefer others similar to, their cagemates.

More recently, Zajonc, Wilson, and Rajecki (1975) have presented data on pecking which indicate that domestic chicks are able to recognize individual strangers and companions as early as $24 \mathrm{~h}$ posthatch, and furthermore, color differences facilitate, but are not necessary for, these discriminations. Evidence was also obtained that close physical interaction contri-

This manuscript is based upon a dissertation submitted by the first author in partial fulfillment of the requirements for the PhD degree. The authors wish to thank Paul B. Siegel for his constructive comments on an early version of this paper. Requests for reprints should be addressed to S. E. Sigman, Department of Psychology, Virginia Polytechnic Institute and State University, Blacksburg, Virginia 24061. butes to and facilitates the identification of individuals. These and other data (Kilham, Klopfer, \& Oelke, 1968; Rajecki, Ivins, \& Rein, 1976) demonstrate the ability of newly hatched chicks to distinguish between conspecifics on the basis of some stimulus characteristic other than color. The particular aspect of their companions which chicks use as a basis for differentiation is not known. The critical factor may be a morphological feature such as size or contour, or it may be, as Zajonc et al. (1975) intimate, differences in the behavior of individuals, such as manner of approach and exploration.

To test these alternate sources of differentiation, the present study examined social discriminations in a situation where either morphological or behavioral differences existed. Specifically, 2-day-old chicks that had been reared in pairs or in isolation were tested for their ability to discriminate between conspecifics which were either familiar or unfamiliar, and which had been, in turn, reared in pairs or in isolation.

\section{EXPERIMENT 1}

\section{Method}

Subjects. White Leghorn chicken eggs were incubated and hatched at the Department of Psychology in incubators maintained at $37.5^{\circ} \mathrm{C}$ and $60 \%-65 \%$ relative humidity. Three days prior to hatching, the eggs were placed on trays in a darkened hatching incubator from which chicks could be removed without lowering the inside temperature or humidity level. As soon as possible after hatching, the chicks were removed from the incubator and housed in a separate room in $18 \times 24 \times 19 \mathrm{~cm}$ cages. The cages provided visual, but not auditory, isolation. Constant illumination in the housing room was provided by overhead fluorescent lights. Temperature in the housing room was maintained at approximately $36^{\circ} \mathrm{C}$. Upon removal from the incubator, the chicks were housed in pairs until a total of 16 pairs were obtained. One member of each pair served as subject, and the other member served as a familiar target. In addition to these 16 pairs, two other pairs of chicks were utilized to serve as unfamiliar targets. 
Apparatus. Testing was conducted in an experimental room separate from the housing room. Temperature in the experimental room was held at a constant $23.5^{\circ} \mathrm{C}$. In each of the experiments, a $55 \times 15 \times 30 \mathrm{~cm} \mathrm{~V}$-maze, painted flat black, was used. Single clear Plexiglas barriers separated the $15-\mathrm{cm}$ deep goalbox from the runway in each arm of the maze. A black guillotine door, located $12.5 \mathrm{~cm}$ from the base of the maze, separated the startbox from the diverging arms. The floor of the apparatus, including the startbox, runways, and goalboxes, consisted of wire mesh attached to the undersides of the apparatus walls. Illumination in the experimental room was provided by a high-intensity lamp (Tensor Model 180) mounted between the arms of the maze so that its beam illuminated the entire apparatus.

Procedure. At $24 \mathrm{~h}$ posthatch, all chicks received a single 5-min apparatus exposure trial (familiarization). During familiarization, each subject's cagemate (familiar target) was placed in one goalbox; the other goalbox remained empty. The empty goalbox was on the left for half of the subjects and on the right for the other half. Each subject was placed in the startbox, and $15 \mathrm{sec}$ later the guillotine door was opened to allow access to both goalboxes. By the end of the familiarization period, all subjects had approached the familiar target (i.e., entered the area $7.5 \mathrm{~cm}$ directly in front of the goalbox) and both chicks were returned to their home cage. Although unfamiliar object birds were also given familiarization in the same manner, the familiarization period was reduced to $2.5 \mathrm{~min}$. At the end of this period, the position of the chicks was reversed and the procedure was repeated, so that each chick spent a total of $5 \mathrm{~min}$ in the apparatus and was exposed to confinement in the goalbox.

To determine preference at $48 \mathrm{~h}$, all subjects were allowed to choose between either the familiar target in one arm of the maze or one of the unfamiliar targets in the other arm. Trials began with the familiar and unfamiliar targets in the goalboxes and the subject in the startbox. After $15 \mathrm{sec}$, the startbox door was opened and the subject was allowed $4 \mathrm{~min}$ in which to approach within $7.5 \mathrm{~cm}$ of the target. Latency to approach and the choice of familiar or unfamiliar target was recorded. Notes of the behavior of all chicks were also made. Once an approach response was made, the subject was allowed $15 \mathrm{sec}$ in the presence of that target, then was returned to its home cage. For half of the subjects, the familiar target was on the left and for half it was on the right. The four unfamiliar target birds were interchanged from subject to subject so that one-fourth of the subjects were exposed to each of the unfamiliar targets.

\section{Results}

Pilot experiments in this laboratory showed that approximately $25 \%$ of chicks exposed to empty goalboxes in the apparatus will approach them. No preference for either arm was evident.

In Experiment 1, all 16 subjects approached targets, with no preference for either target bird. Seven subjects approached the familiar target and 9 subjects approached the unfamiliar target. Latency to approach familiar targets $(\bar{X}=23.9)$ did not reliably differ from latency to approach unfamiliar targets $(\bar{X}=49.7$; $\mathrm{U}=21, \mathrm{p}>.05)$.

When the startbox door was opened, both targets typically reacted in the same manner. Distress vocalizations, often emitted before the door was opened, ceased and were replaced by pleasure calls. Target birds then lowered their heads, pressed their bodies against the Plexiglas barrier, and moved rapidly from side to side in an apparent effort to push through the Plexiglas and approach the subject. Occasionally, targets pecked at the barrier or at other parts of the goalbox, creating a tap readily audible to the experimenter. The subjects typically emitted distress calls until the startbox door was opened, then began to emit pleasure calls. There was a typical momentary hesitation after the startbox door was opened, then the subject advanced to the target, lowered its head, and pressed against the Plexiglas barrier.

\section{EXPERIMENT 2}

From the first experiment, it is evident that socially reared chicks do not prefer familiar socially reared chicks to unfamiliar socially reared chicks. However, since the behavior of both target chicks was essentially the same, i.e., "social," it is possible that the chicks simply do not discriminate individuals whose behavior is similar. For that reason, the second experiment was designed to determine if socially reared chicks prefer socially reared chicks to isolationreared chicks. In this experiment, socially reared chicks were given the choice of either unfamiliar targets raised in pairs or unfamiliar targets raised in isolation.

\section{Method}

Seven pairs of chicks served as subjects. In addition to these 14 chicks, four additional pairs served as unfamiliar social targets, while 7 chicks, individually housed, were unfamiliar isolate targets. Familiarization at $24 \mathrm{~h}$ was conducted as in Experiment 1. Members of subject pairs were placed in the apparatus simultaneously, one in the startbox and one in the goalbox. After $2.5 \mathrm{~min}$, the positions of the chicks were reversed and the procedure was repeated. Social targets were familiarized in the same fashion. Isolate targets were placed individually in the goalbox, the startbox door was opened, and after $5 \mathrm{~min}$ the chick was removed. At $48 \mathrm{~h}$, all subjects were tested for preference. The small number of target birds made it necessary to use the same targets for more than one subject, but the same two targets were always paired when used a second time. Thus, Subjects 1 and 2 had the same targets, Subjects 3 and 4 had the same targets, and so forth.

\section{Results}

As was the case in Experiment 1, social targets typically emitted pleasure calls, lowered their heads, and pressed against the Plexiglas barrier when the startbox door was opened. In contrast to this, the isolate targets typically stood facing the wall in the back of the goalbox, occasionally moving slowly around the box, emitting distress calls. Unlike the social targets, which frequently pecked at parts of the apparatus, the isolate birds rarely pecked. Subjects typically reacted to the opening of the startbox door as in Experiment 1.

Twelve of the 14 subjects exhibited a preference for the social target (two-tailed binomial test, $\mathrm{p}=.012$ ). Mean latency to approach social targets and isolate targets was 7.9 and $9.6 \mathrm{sec}$, respectively, and did not differ statistically. 


\section{EXPERIMENT 3}

It appears from the first two experiments that, while socially reared chicks do not discriminate in approaching socially reared targets, they do discriminate in approaching socially and nonsocially reared targets. The third experiment was designed to determine if chicks reared in isolation also discriminate between social chicks and isolate chicks. More specifically, chicks reared in isolation were given a choice between unfamiliar targets reared in pairs and unfamiliar targets reared in isolation.

\section{Method}

Eight chicks from each of two weekly hatches were housed in isolation. In addition, four pairs of chicks and eight isolate chicks were taken from each weekly hatch to serve as social targets and isolate targets, respectively. Familiarization for subjects was accomplished at $24 \mathrm{~h}$ by placing them in the empty apparatus. Familiarization for social and isolate targets was accomplished as in the previous experiments. Testing at $48 \mathrm{~h}$ proceeded as in Experiment 2 except that targets were not used for more than one subject.

\section{Results}

Isolate subjects exhibited a preference for social targets (two-tailed binomial test, $p=.036$ ). One sub. ject did not approach a target and was omitted from statistical analysis. Twelve of the remaining 15 subjects preferred the social target. No reliable differences were found in latency to approach. Mean latency to approach the social target was $90.4 \mathrm{sec}$, and the mean latency to approach for those three subjects that chose the isolate target was $116.1 \mathrm{sec}$. The approach latencies are notably longer than in the previous experiments, but are not surprising since the previous experiments used socially reared subjects and the present experiment used isolate subjects.

It is evident from the longer latency scores that when the door was opened subjects typically stood at the back of the startbox longer than in previous experiments. Also, in contrast to the previous experiments, subjects continued to emit distress calls after the startbox door was opened. Once an approach response was begun, however, the subjects' behavior invariably resembled that of subjects in the previous experiments. Isolate targets typically acted as isolate targets previously, but some isolate targets began to act in a manner similar to that of social targets before the trial was completed. Undoubtedly this is because the subjects were taking longer to approach, and isolate targets therefore were receiving more exposure to them. Social targets also did not act as previous social targets had acted. Distress calls did not terminate with the lifting of the startbox door, and only after considerable delay did social targets lower their heads, press against the Plexiglas, and emit pleasure calls. The results of the second experiment suggest that this may be due to social targets' ability to dis- criminate between the isolate subjects and the chicks with whom they were reared. This, too, probably enters into the longer latency scores.

\section{DISCUSSION}

The present experiments reveal that chicks 2 days old discriminate and preferentially respond to conspecifics in the absence of strain or color differences, thus confirming the results of Rajecki et al. (1976) and Zajonc et al. (1975). The discrimination appears to be based upon differences in behavior between the two targets, and furthermore, prior social experience is not necessary for the discrimination to occur. The first experiment demonstrates that chicks with similar behaviors are not preferentially approached by other chicks, even though the morphological differences that exist between the targets according to Zajonc et al. (1975) are sufficient to allow discrimination by companions. Perhaps the subjects are able to distinguish between the two targets but do not have a preference for one over the other. From the second experiment, it is clear that chicks whose behaviors are dissimilar are discriminated and socially reared targets are preferentially approached. The same morphological differences exist between targets as in the first experiment, but here the behaviors also differ. The third experiment demonstrates that prior social experience on the part of the subject is not a prerequisite for such preferences. In the present case at least, the subject chicks did not simply imprint upon and develop a preference for the behavior of their cagemate.

Since the only systematic difference between targets in all of the experiments was the differential rearing conditions, it is clear that the targets' prior social experience is the underlying factor in the subjects' preferential approaches. Those preferential approaches may reflect an attraction to the social target, a withdrawal from the isolate target, or both. This approachavoidance confound is always an inherent part of the two-choice situation employed.

Results of Experiments 1 and 2 suggest that behavioral features may be more important in determining approach pereferences than are physical characteristics such as size, shape, or color. Recent evidence has supported this hypothesis (Sigman \& Schulman, Note 1).

It is not clear which component of the targets' behavior is the functional determinant of choice. Chicks generally have been found to approach moving objects more readily than stationary ones (Hess, 1973, p. 115), and Hoffman and Ratner (1973) have referred to movement as a releaser of filial behavior. But, even though the isolate targets were relatively inactive, they were by no means immobile and it seems likely that other components of the targets' behavior are also important in determining approach. 
Several possible alternatives are obvious. One of these is the different vocalizations of the social and isolate targets. The social targets tended to emit contentment calls, while the isolate targets tended to emit distress calls, a finding in agreement with Rajecki et al. (1976). It is feasible that distress and contentment calls are differentially attractive to chicks. If so, vocalizations may be a discriminandum used by the subjects to determine approach. An investigation of the relative attractiveness of the two types of vocalizations is being conducted by Elizabeth D. Robinson-Guy in our laboratory. An alternative to the vocalization preference is that the particular posture adopted by the social target, namely lowering the head and a rapid side-to-side motion, is attractive to other chicks. Still another possibility lies in the differential rates of environmental pecking. Social targets were observed to engage in considerably higher rates of pecking than were the isolate targets. It is possible that the subject chicks were attracted to the pecking of the targets, either because of the pecking motions perse or because of the audible tap produced.

Gray (1961) has argued that Leghorn chicks up to 2 days old show a preference for an agemate as opposed to a hen, and suggests that it is advantageous for chicks of a brood to remain together and not attempt to follow the mother until they have gained sufficient locomotor abilities. If such is the case, data presented here suggest that part of such group cohesion is a function of the manner in which the chicks interact. Not only are physical instigators of approach continually strengthening the unity of the brood by attracting the chicks to each other, but behavioral instigators are also operating to insure the survival of the brood as a unit. A sick or otherwise incapacitated chick may not provide the behavioral component that would continue to attract other chicks to it, or it may include a component that repels other chicks, thus insuring that strong, healthy birds will not linger with the infirm and be left by the group.

To summarize, it is clear that Leghorn chicks respond to others on the basis of behavior and that the approach response of these chicks may be more complicated than a direct reaction to the physical characteristics of a living target. It has been suggested that behavioral features may be more important than morphological features in determining approach preferences in some situations. Furthermore, it is not necessary that chicks be exposed to the particular behavior in order to demonstrate a preference for it. Such behavior preferences may be a contributing factor to brood unity among chicks.

\section{REFERENCE NOTE}

1. Sigman, S. E., \& Schulman, A. H. A comparison of morphological and behavioral factors underlying social preference. Manuscript in preparation.

\section{REFERENCES}

GraY, P. H. The releasers of imprinting: Differential reactions to color as a function of maturation. Joumal of Comparative and Physiological Psychology, 1961, 54, 597-601.

GuHl, A. M. The development of social organization in the domestic chick. Animal Behaviour, 1958, 6, 92-110.

Hess, E. H. Imprinting: Early experience and the psychobiology of attachment. New York: Van Nostrand, 1973.

Hoffman, H. S., \& Ratner, A. M. A reinforcement model of imprinting. Psychological Review, 1973, 80, 527-544.

Kilham, P., Klopfer, P. H., \& OElKe, H. Species identification and color preferences in chicks. Animal Behaviour, 1968, 16, 238-244.

RAJECK, D. W., Ivins, B., \& REIN, B. Social discrimination and aggressive pecking in domestic chicks. Joumal of Com. parative and Physiological Psychology, 1976, 90, 442-452.

RAECKI, D. W., \& LAKE, D. Social preference in chicks as a function of own color and rearing condition. Revue de Comportement Animal, 1972, 6, 151-156.

Salzen, E. A., \& Cornell, J. M. Self-perception and species recognition in birds. Behaviour, 1968, 30, 44-65.

Schuelderup-EbBe, T. Social behavior in birds. In C. Murchison (Ed.), Murchison's handbook of social psychology. Worcester: Clark University Press, 1935.

Smrth, W. Social 'learning' in domestic chicks. Behaviour, $1957,11,40-55$.

Zajonc, R. B., Wilson, W. R., \& RAJecki, D. W. Affiliation and social discrimination produced by brief exposure in day-old domestic chicks. Animal Behaviour, 1975, 23, 131-138.

(Received for publication April 5, 1977; revision accepted November $7,1977$. ) 